\title{
Ultra-low-dose chest CT performance for the detection of viral pneumonia patterns during the COVID-19 outbreak period: a monocentric experience
}

\author{
Joël Greffier ${ }^{1}$, Adel Hoballah ${ }^{1}$, Alexandre Sadate ${ }^{1}$, Fabien de Oliveira ${ }^{1}$, Pierre-Geraud Claret ${ }^{2}$, \\ Hélène de Forges ${ }^{1}$, Paul Loubet ${ }^{3}$, Jean-Marc Mauboussin ${ }^{3}$, Aymeric Hamard ${ }^{1}$, Jean-Paul Beregi ${ }^{1}$, \\ Julien Frandon ${ }^{1}$
}

${ }^{1}$ Department of Medical Imaging, Nîmes University Hospital, University of Montpellier, Medical Imaging Group Nîmes, Nîmes, France; ${ }^{2}$ Department of Anesthesia Resuscitation Pain Emergencies, Nîmes University Hospital, University of Montpellier, Nîmes, France; ${ }^{3}$ VBMI, INSERM U1047, Department of Infectious and Tropical Disease, CHU Nîmes, Univ Montpellier, Nîmes, France

Correspondence to: Julien Frandon. Department of Medical Imaging, Nîmes University Hospital, University of Montpellier, Medical Imaging Group Nîmes, EA 2415, Bd Prof Robert Debré, 30029 Nîmes Cedex 9, France. Email: julien.frandon@chu-nimes.fr.

Background: Ultra low dose chest computed tomography (CT) acquisitions have been used for selected emergency room patients with acute dyspnea or minor thoracic trauma. The purpose of this study was to evaluate the diagnostic performance of ultra-low-dose (ULD) chest CT for detecting viral pneumonia patterns compared to standard (STD) dose chest CT.

Methods: All consecutive adult patients with two non-enhanced chest CT acquisitions, one STD and one ULD, for suspicion of viral pneumonia between March $5^{\text {th }}$ and April $2^{\text {nd }} 2020$ were included. CT results were divided into two groups: non-viral pneumonia CT or compatible with viral pneumonia CT based on viral pneumonia CT patterns: ground-glass opacity (GGO), consolidation, crazy paving, air bronchogram signs and fibrous stripes. The diagnostic performance of ULD CT for suspicion of viral pneumonia was evaluated. For CTs compatible with viral pneumonia, CT pattern detection on ULD CT was assessed and STD CT was used as a reference.

Results: The study included 380 patients with 97 CTs $(25.5 \%)$ compatible with viral pneumonia. The mean effective doses (EDs) were $1.66(1.29 ; 2.18) \mathrm{mSv}$ for STD and $0.20(0.18 ; 0.22) \mathrm{mSv}$ for ULD CT $(\mathrm{P}<0.001)$. The sensitivity and specificity of ULD CT for viral pneumonia detection were $98.9 \%$ and $99.0 \%$, respectively. GGO, consolidation and fibrous stripes were equally visible in STD and ULD in 100\% (n=97), $36 \%(n=35)$ and $23 \%(n=22)$ of compatible viral pneumonia-CT patients, respectively. Air bronchogram sign detection was equivalent, concerning $23 \%(n=22)$ of patients in STD and 22\% $(n=21)$ in ULD. Crazy paving was visible in $24 \%(n=23)$ of patients in STD and only $8 \%(n=8)$ in ULD ( $P=0.003)$.

Conclusions: In comparison to STD dose chest CT, ULD chest CT, with a mean reduction dose of $88.0 \%$, has comparable diagnostic performance for detecting viral pneumonia on CT.

Keywords: Multidetector computed tomography (multidetector CT); ultra-low-dose CT (ULD CT); coronavirus infections

Submitted Oct 21, 2020. Accepted for publication Feb 18, 2021.

doi: 10.21037 /qims-20-1176

View this article at: http://dx.doi.org/10.21037/qims-20-1176 


\section{Introduction}

Human coronaviruses are considered as important pathogens causing upper and lower respiratory tract infections such as pneumonia and even acute respiratory distress syndrome (1). SARS coronavirus was identified as a member of the Coronaviridae family in late 2003 after a worldwide epidemic. Other coronaviruses such as the Middle East Respiratory Syndrome have been associated with regional outbreaks in the past and could re-emerge to produce outbreaks in the future (2). The current outbreak of the novel SARS-CoV-2 coronavirus, responsible for COVID-19 disease, with its epicenter initially in the Hubei Province of China, has now spread to many western countries. The World Health Organization (WHO) announced the COVID-19 outbreak as a pandemic (3).

Diagnosis relies on the identification of viral ribonucleic acid (RNA) by reverse-transcription polymerase chain reaction (RT-PCR). The RT-PCR test, not always available in many countries or centers, has been shown to have moderate sensitivity with delayed positivity and therefore confirmed COVID-19 diagnosis may be missed (4). A large series of 1,014 Chinese patients showed a higher sensitivity for chest computed tomography (CT) as compared to RTPCR, with a mean time interval between the initial negative to positive RT-PCR results of $5.1 \pm 1.5$ days (5).

Characteristic CT findings specific to COVID-19 have been described in the recent literature with mainly bilateral ground-glass opacities (GGOs) and a predominantly peripheral, subpleural location (6-9). Some authors have suggested using CT-scans as a screening tool (4). However, these CT characteristics have moderate sensitivity for distinguishing COVID-19 pneumonia from other viral pneumonia with the same CT patterns $(10,11)$. CTscanning has also shown its usefulness for the follow-up of COVID-19 patients, especially revealing lung imaging changes during the course of the disease $(7,12)$. The severity of lung abnormalities on CT has also been reported as a predictive factor of mortality (13).

Altogether, CT-scanning seems to have an important role to play in these viral pneumonia outbreak periods with rapid detection of lung damage, which can help to control potential transmission early on. Due to limited access to RTPCR tests and their moderate sensitivity, many patients are addressed to the emergency department for a CT-scan (14). Its widespread implementation warrants a critical review of the radiation dose. Some authors have suggested using an ultra-low-dose (ULD) CT-scan for suspected
COVID-19 patients to efficiently decrease the radiation dose delivered $(15,16)$. However, these studies have not demonstrated the diagnostic performance of ULD CT for detecting viral pneumonia patterns on $\mathrm{CT}$ as compared to standard (STD) chest CT acquisitions $(15,16)$. The use of ULD CT acquisitions for chest-located lesions has been routine clinical practice since 2016 at our Institute for patients with acute dyspnea or minor blunt thoracic trauma $(17,18)$.

The aim of the present study was to evaluate the diagnostic performance and diagnostic confidence level of ULD CT acquisition compared to the STD acquisition for diagnosing viral pneumonia in the context of the COVID-19 outbreak. Secondary objectives were to assess, both objectively and subjectively, the image quality and accuracy of the diagnosis of viral pneumonia patterns especially GGOs with ULD CT acquisition.

\section{Methods}

The study was conducted in accordance with the Declaration of Helsinki (as revised in 2013) and approved by the institutional review board of the Nîmes University Hospital. The protocol was in accordance with the clinical practices decided in our department to face the pandemic situation. The individual informed consent requirement was waived by the IRB because of the retrospective character of the analysis.

\section{Patients}

All consecutive patients who underwent chest CT from March $5^{\text {th }}$ to April $2^{\text {nd }} 2020$ and had been admitted to the COVID-19 patient care pathway at our public university hospital were included. Patients under 18 years of age or for whom the two CT acquisitions, STD and ULD, had not been performed, were not included.

\section{Chest CT protocol}

Patients lay in a supine position during a non-enhanced consecutive acquisition CT protocol (STD followed by ULD). ULD CT acquisition as a replacement for chest $\mathrm{X}$-ray was already used at the emergency radiological department in our Institution for patients with acute dyspnea or minor blunt thoracic trauma $(17,18)$.

Acquisitions were performed on a Somatom EDGE (Siemens Healthineers) with a physical beam collimation 
of $64 \times 0.6 \mathrm{~mm}$ and rotation time of $0.288 \mathrm{~s} /$ rot. For STD acquisition, the acquisition parameters were as follows: tube voltage of reference $120 \mathrm{kVp}$, tube current of reference $61 \mathrm{mAs}$, pitch factor of 1.2. The automatic tube current modulation (CareDose 4D) system was used and the automatic tube voltage selection (Care $\mathrm{kV}$ ) was activated on the "non-enhanced" setting. For ULD acquisition, the dataset was obtained with a tube voltage of $100 \mathrm{kVp}$ [no activated Care $\mathrm{kV} ; 120 \mathrm{kVp}$ for body mass index (BMI) $>35 \mathrm{~kg} / \mathrm{m}^{2}$ ], a $10 \mathrm{~mA}$ tube current (without CareDose 4D) and a 1.7 pitch.

For both protocols, raw data were reconstructed using the "moderately smooth" (I30f, mediastinal) reconstruction kernel and a slice thickness of $1 \mathrm{~mm}$ ( $0.7 \mathrm{~mm}$ overlapped). For the STD protocol, images were obtained using Level 3 of ADMIRE with the "very strong" (I70f, lung images) reconstruction kernel. To reduce the image noise, the Level 4 of ADMIRE with the "moderately strong" (I50f, lung images) reconstruction kernel was used for the ULD protocol. All these parameters correspond to those usually used in our Institution for both STD and ULD chest CT.

\section{CT imaging: review and findings}

According to the viral pneumonia characteristics described in the literature, a senior radiologist, R1, with 15 years' experience of cardiothoracic imaging (JF) reviewed all STD CT-scans to classify CT images into two groups: non-viral pneumonia CTs (normal CT, non-viral pneumonia, and others diseases) and CTs compatible with viral pneumonia [peripherally distributed, multifocal or patchy, GGOs; patchy consolidations; fibrous stripes (7), crazy paving (6)], and white lung $(4,11)]$. This classification was used as the reference.

$\mathrm{R} 1$, who routinely used ULD protocols and R2 (AH) who had never interpreted on ULD CT, both blinded to the CT acquisition doses, the diagnosis decision taken during care, independently of the study, to the reference classification and to the other reader's evaluation, both reviewed all CT images, STD and ULD, in a random order. They divided CT images into two groups according to the above-mentioned classification. Main viral pneumonia patterns such as GGO, consolidation, fibrous stripes and crazy paving were also reported by the two readers on STD and ULD acquisitions.

\section{Objective image quality}

Objective assessments of image quality were performed for all patients in the compatible viral pneumonia CT group. For each patient, four ROIs of approximately $0.7 \mathrm{~cm}^{2}$ were placed on lung images: in the trachea, in the normal pulmonary parenchyma, on the GGO and, if present, on consolidation. Only one ROI was computed per pattern, and only if the given pattern had an area greater than $0.5 \mathrm{~cm}^{2}$. In each ROI, the mean $\left(\mathrm{N}_{\mathrm{CT}}\right)$ and the standard deviation (noise) of pixel values were computed.

The contrast-to-noise ratio (CNR) was calculated for each tissue assessed using the $\mathrm{N}_{\mathrm{CT}}$ and image noise measured within the ROI of trachea as reference, as follows:

$$
C N R=\frac{\left|N_{C T, \text { tissue }}-N_{C T \text {,trachea }}\right|}{\sqrt{\frac{\left(\sigma_{\text {tissue }}^{2}+\sigma_{\text {trachea }}^{2}\right)}{2}}}
$$

\section{Subjective image quality assessment}

Subjective image quality assessment was performed for all patients by R1 and R2. The image quality of diagnostic findings on chest CT images was rated according to a 5-point Likert scale (17): 1 point: excellent, 2 points: good, 3 points: acceptable, 4 points: sub-optimal, 5 points: unacceptable. The radiologists also expressed a subjective degree of diagnostic confidence as "certain" or "uncertain".

\section{Dosimetry evaluation}

The CT dose index volume $\left(\mathrm{CTDI}_{\mathrm{vol}}\right)$ and the dose length product (DLP) were retrieved from the review report available in the CT workstation at the end of the acquisitions. The effective dose (ED) was calculated for each CT examination by multiplying the DLP by the specific chest conversion coefficient $(0.014 \mathrm{mSv} / \mathrm{mGy} / \mathrm{cm})(19)$.

\section{Statistical analyses}

Statistical analyses were carried out using $\mathrm{R}$ open source software (v3.20) and the RStudio editing tool (v0.98.113). Using the diagnosis provided on STD, sensitivity and specificity of ULD protocol were calculated with a $95 \%$ confidence interval for each reader: junior and senior. Data $\left(\mathrm{CTDI}_{\mathrm{vol}}\right.$, DLP, E, mean attenuation, CNR and 
Table 1 Demographic data and clinical symptoms

\begin{tabular}{lccc}
\hline Factor & All $(\mathrm{n}=380)$ & Non-viral pneumonia CT $(\mathrm{n}=283)$ & Compatible viral pneumonia CT $(\mathrm{n}=97)$ \\
\hline Demographic & & & $66.4 \pm 15.4$ \\
Age (years) & $66.3 \pm 18.7$ & $66.9 \pm 19.1$ & $44(45.4)$ \\
Sex male, $\mathrm{n}(\%)$ & $195(48.7)$ & $151(53.4)$ & $26.8 \pm 5.7$ \\
BMl $\left(\mathrm{kg} / \mathrm{m}^{2}\right)$ & $27.4 \pm 8.5$ & $28.3 \pm 9.1$ & $33(34.0)$ \\
Clinical symptoms, $\mathrm{n}(\%)$ & & & $63(64.9)$ \\
Asthenia & $72(18.9)$ & $39(13.8)$ & $10(10.3)$ \\
Cough & $224(58.9)$ & $161(56.9)$ & $61(62.9)$ \\
Chest pain & $53(13.9)$ & $43(15.2)$ & $71(73.2)$ \\
Dyspnea & $232(61.1)$ & $171(60.4)$ & $25(25.8)$ \\
Fever & $274(72.1)$ & $203(71.7)$ & $11(11.3)$ \\
O requirement & $116(30.5)$ & $91(32.2)$ & $19(6.7)$ \\
Respiratory distress & $30(7.9)$ & & \\
\hline
\end{tabular}

BMI, body mass index; CT, computed tomography.

image noise) were tested for normal distribution using the Shapiro-Wilk test. For all continuous variables, statistics were reported as means \pm standard deviation or as medians $\left(1^{\text {st }}\right.$ quartile; $3^{\text {rd }}$ quartile), as appropriate. The Student $t$-test for independent samples and the paired Mann-Whitney $U$ test were used to compare variables between the ULD and STD protocols. A P value lower than 0.05 was considered significant. For a given CT protocol, the agreement coefficient between the two radiologists was computed with Cohen's kappa test and classified as poor $(\kappa=0.00-0.20)$, fair $(\kappa=0.21-0.40)$, moderate $(\kappa=0.41-0.60)$, $\operatorname{good}(\kappa=0.61-0.80)$, or excellent ( $\kappa=0.81-1.00)$. The paired Mann-Whitney $U$ test was used to assess the inter-protocol agreement for each radiologist and to compare CT findings between protocols.

There was no missing data and no patients were excluded from the analysis after their initial inclusion. No adverse events occurred due to chest CT examinations.

\section{Results}

\section{Patient demographics and pathology findings}

During the study period, 447 chest CT examinations were performed on suspected COVID-19 patients, 67 of whom were excluded from the analysis ( $\mathrm{n}=63$, no ULD acquisition, $\mathrm{n}=4$, patient $<18$ years old). Finally, 380 patients (195 men and 185 women) were included (Table 1), with a mean age of $66.3 \pm 18.7$ years old $(68.2 \pm 16.0$ for men and $64.2 \pm 21.1$ for women). The mean BMI was $27.4 \pm 8.5 \mathrm{~kg} / \mathrm{m}^{2}(26.5 \pm 7.5$ for men and 28.5 \pm 9.6 for women).

Among the 380 patients, 97 patients (26\%) were considered to have CTs compatible with viral pneumonia. For these 97 patients, GGOs were found on STD images for $100 \%$ of cases, fibrous stripes for $23 \%$, consolidation for $36 \%$, crazy paving for $24 \%$ and air bronchogram signs for $23 \%$ (Figure 1). Similar results were found for ULD, except for the crazy paving, which was only detected in 8 out of 24 patients $(\mathrm{P}<0.05)$ (Figure 2).

\section{Sensitivity and specificity}

For R1, the ULD CT sensitivity was $98.9 \%(93.5 \%$; $100.0 \%)$ and the specificity was $99.0 \%(96.8 \% ; 99.8 \%)$ and for R2 sensitivity was $98.9 \%(93.5 \% ; 100.0 \%)$ and specificity was $97.6 \%$ (94.9\%; 98.9\%), respectively (Table 2).

\section{Objective image quality}

The mean attenuation was significantly different with ULD and STD (Table 3) for trachea $(\mathrm{P}<0.001)$ and normal lung $(\mathrm{P}<0.01)$ but not significant for GGO and consolidation. Image noise and CNR were significantly better with the ULD protocol than with the STD protocol for all assessed tissues $(\mathrm{P}<0.001)$. The image noise decrease varied from $-25 \%$ for trachea to $-43 \%$ for consolidation. The CNR increase varied from $52 \%$ for GGO to $58 \%$ for normal lung. 

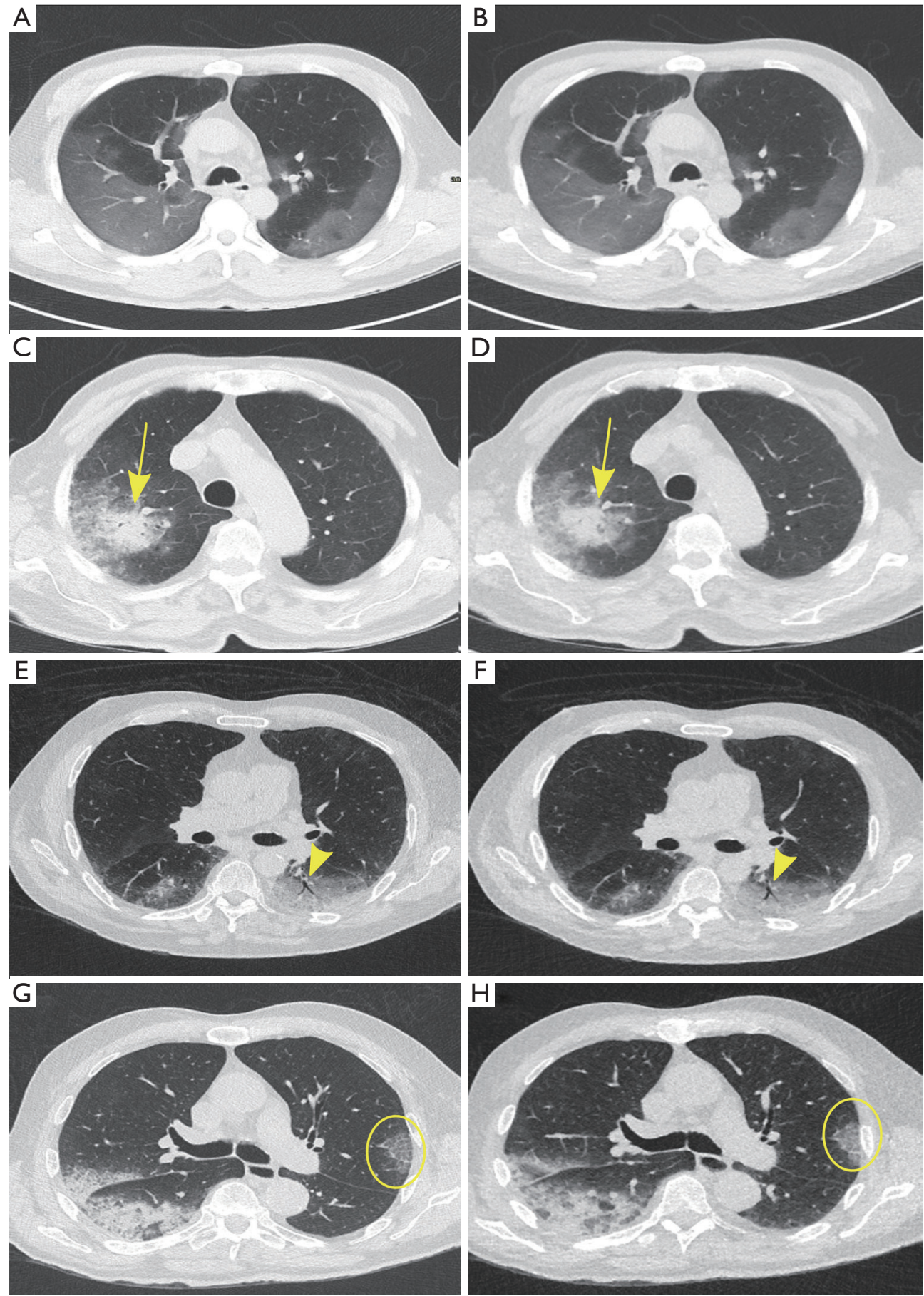

Figure 1 Different patterns compatible with viral pneumonia in patients on STD (left) and ULD (right) acquisitions at the same anatomical position. (A,B) show extensive subpleural ground; (C,D) consolidation (yellow arrow) surrounded by ground glass; (E,F) air bronchogram (yellow arrow head), equally visible on STD and ULD acquisitions; $(\mathrm{G}, \mathrm{H})$ depict crazy paving (yellow circle), not clearly identifiable on the ULD acquisition. STD, standard; ULD, ultra-low-dose. 


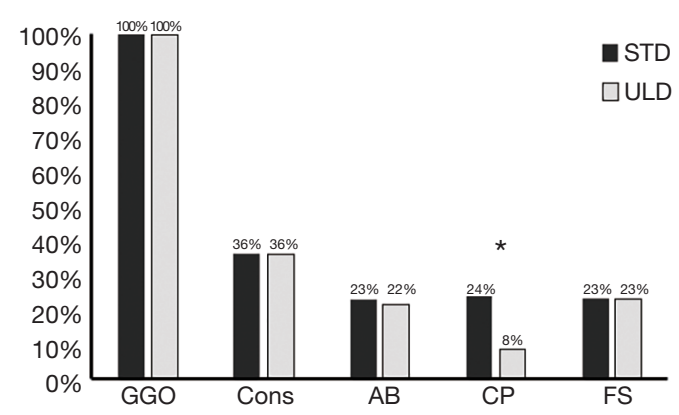

Figure 2 Distribution of findings for ULD and STD chest CTs. *, P value lower than 0.05 . ULD, ultra-low-dose; STD, standard; CT, computed tomography; GGO, ground-glass opacity; Cons, consolidation; $\mathrm{AB}$, air bronchogram; $\mathrm{CP}$, crazy paving; FS, fibrous stripes.

\section{Subjective image quality}

Both radiologists (R1 and R2) mainly rated the diagnostic image quality of the STD acquisition as excellent: $93 \%$ were "excellent" for R1 and 95\% "excellent" for R2 (Table 4). For the ULD images, R1 scored "excellent" in $71 \%$ of cases, good in $26 \%$ and acceptable in $3 \%$ and R2 ranked $65 \% / 30 \% / 5 \%$, respectively. The inter-observer agreement was good for both the STD $(k=0.86)$ and ULD $(\mathrm{k}=0.87)$ protocols. The inter-protocol comparison was significantly different for the two radiologists $(\mathrm{P}<0.001)$.

$\mathrm{R} 1$ rated his diagnostic confidence level as "certain" in 99.5\% of cases for the STD images and 99\% for the ULD images. For R2, the diagnostic confidence level was "certain" in $98 \%$ of cases for the STD acquisition and in 91\% of cases for the ULD acquisition. The inter-observer agreement was fair $(k=0.40)$ for the STD protocol and poor $(k=0.18)$ for ULD. The inter-protocol comparison was not significantly different for $\mathrm{R} 1$ but significantly different for $\mathrm{R} 2(\mathrm{P}<0.001)$.

\section{Dosimetry}

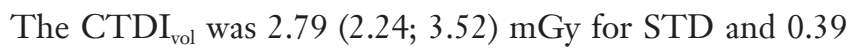
(0.39; 0.40) mGy for ULD. The DLP was 118.6 (92.2; 155.6) $\mathrm{mGy} \cdot \mathrm{cm}$ and the ED was $1.66(1.29 ; 2.18) \mathrm{mSv}$ for STD as compared to a DLP of $14.2(13.1 ; 15.4) \mathrm{mGy} \cdot \mathrm{cm}$ and $\mathrm{ED}$ of $0.20(0.18 ; 0.22) \mathrm{mSv}$ for ULD. All these parameters were significantly different $(\mathrm{P}<0.001)$ between the two CT protocols. In addition, using the Care $\mathrm{kV}$ for the STD chest protocol, $120 \mathrm{kVp}$ were used for 276 patients (73\%), $100 \mathrm{kVp}$ for 100 patients (26\%) and $140 \mathrm{kVp}$ for 4 patients $(1 \%)$.

\section{Discussion}

This study demonstrated a high diagnostic performance and level of confidence of ULD chest CT compared to STD CT acquisition for suspicion of viral pneumonia on CT. Sensitivity, specificity and diagnostic confidence level were similar for the STD and ULD protocols, except for the crazy paving pattern.

The highest values of sensitivity and specificity found were in the same range for both acquisitions and were both close to those previously published for the specific diagnosis of COVID-19 viral pneumonia $(4,6,7)$. The lung image quality obtained with the ULD CT acquisition was suitable for detecting GGOs, fibrous stripes, consolidation and air bronchogram signs, all patterns leading to suspicion of viral pneumonia on CT. However, the change in image texture for ULD lung images was not suitable for detecting crazypaving patterns. Crazy-paving patterns are characterized by scattered or diffuse GGOs with superimposed interlobular septal thickening and intralobular lines. This damage to the interstitial space is a common finding on thin-section CT for the lungs (20). Therefore, ULD acquisition might not be useful for the long-term follow-up of viral pneumonia patients for whom lung fibrosis is suspected.

The ED for ULD acquisition was close to $0.2 \mathrm{mSv}$ and $88 \%$ lower than that of STD acquisition. The dose level for the ULD CT protocol was similar to that previously published for patients with acute dyspnea or minor blunt thoracic trauma $(17,18)$. For the currently reported STD acquisition, the dose level was higher than what was published in 2016. Indeed, we used the STD protocol designed for the diagnosis of interstitial lung diseases with an approximately $20 \%$ higher dose level than previously published and considered as low-dose CT acquisition. The STD and ULD dose levels reported here were similar to those recommended by Kang et al. (15) for the detection and management of COVID-19 patients, and lower than those published by Agostini et al. (16) for 10 patients with COVID-19. The ULD dose levels in these studies were defined for a dual-source CT-scan using specific tools such as tin filter or high-pitch, which limits its use to a limited number of CT-scans. In our study, the ULD dose level was defined on a single source CT-scan with classical acquisition and reconstruction parameters and can therefore be adapted to most modern single-source CT-scans with an iterative reconstruction algorithm.

The results of the objective image quality assessment showed that, for lung imaging, image noise decreased and 
Table 2 Sensitivity, specificity, positive predictive value and negative predictive value of ULD chest CT acquisitions obtained by the senior (radiologist 1) and junior (radiologist 2) radiologists for viral pneumonia detection

\begin{tabular}{lcc}
\hline ULD performance & Radiologist 1 & Radiologist 2 \\
\hline Sensitivity $(95 \% \mathrm{Cl})$ & $98.9 \%(93.5 \% ; 100.0 \%)$ & $98.9 \%(93.5 \% ; 100.0 \%)$ \\
Specificity $(95 \% \mathrm{Cl})$ & $99.0 \%(96.8 \% ; 99.8 \%)$ & $97.6 \%(94.9 \% ; 98.9 \%)$ \\
Positive predictive value $(95 \% \mathrm{Cl})$ & $96.9 \%(93.4 \% ; 100.0 \%)$ & $92.9 \%(87.9 \% ; 98.0 \%)$ \\
Negative predictive value $(95 \% \mathrm{Cl})$ & $99.6 \%(99.0 \% ; 100.0 \%)$ & $99.6 \%(98.9 \% ; 100.0 \%)$ \\
\hline
\end{tabular}

Outcomes obtained from STD chest CT acquisitions were used as reference. ULD, ultra-low-dose; STD, standard; CT, computed tomography.

Table 3 Mean attenuation, image noise and CNR measured on different anatomical structures on ULD and STD chest CT acquisitions

\begin{tabular}{|c|c|c|c|c|}
\hline Objective image quality parameters & STD & ULD & Mean difference (\%) & $P$ value \\
\hline \multicolumn{5}{|l|}{ Mean attenuation $\left(\mathrm{N}_{\mathrm{CT}}, \mathrm{HU}\right)$} \\
\hline Trachea & $-926.5(-939.0 ;-914.0)$ & $-940.0(-948.8 ;-925.5)$ & 1 & $\mathrm{P}<0.001$ \\
\hline Normal lung & $-861.0(-879.0 ;-843.0)$ & $-865.5(-882.8 ;-842.8)$ & 1 & $\mathrm{P}<0.01$ \\
\hline GGO & $-479.0(-543.0 ;-425.3)$ & $-475.5(-527.8 ;-413.5)$ & -1 & 0.26 \\
\hline \multicolumn{5}{|l|}{ Image noise (HU) } \\
\hline Trachea & $71.5(64.5 ; 81.9)$ & $54.3(48.9 ; 59.3)$ & -25 & $\mathrm{P}<0.001$ \\
\hline Normal lung & $94.9(85.5 ; 110.8)$ & $63.9(58.3 ; 73.7)$ & -33 & $\mathrm{P}<0.001$ \\
\hline GGO & $156.0(143.3 ; 176.5)$ & $101.5(87.3 ; 115.0)$ & -35 & $\mathrm{P}<0.001$ \\
\hline Normal lung & $0.71(0.49 ; 0.93)$ & $1.07(0.76 ; 1.34)$ & 58 & $P<0.001$ \\
\hline GGO & $3.16(2.78 ; 3.76)$ & $4.80(4.11 ; 5.89)$ & 52 & $\mathrm{P}<0.001$ \\
\hline Condensation & $6.20(5.36 ; 7.39)$ & $10.35(8.78 ; 11.71)$ & 57 & $P<0.001$ \\
\hline
\end{tabular}

Outcomes of mean difference of each metric between both chest CT protocols and its respective statistical results are depicted. Values are expressed as medians ( $1^{\text {st }}$ quartile; $3^{\text {rd }}$ quartile). A $P$ value lower than 0.05 was considered significant. CNR, contrast-to-noise ratio; ULD, ultra-low-dose; STD, standard; CT, computed tomography; $\mathrm{N}_{\mathrm{CT}}$, mean attenuation of pixel; HU, Hounsfield unit; GGO, ground-glass opacity.

CNR increased with the ULD CT acquisition as compared to the STD CT acquisition. Similar changes in noise and CNR were observed in normal lung and in pathological areas (GGO and consolidation). These changes were related to the increase in the iterative level and the use of a softer reconstruction kernel. However, these modifications changed the image texture, increasing image smoothness and reducing spatial resolution (21-23). Similar results were found by Macri et al. on 133 consecutive dyspneic patients but with lower variations in image noise and CNR (17). These differences are explained by the fact that we used a newer single-source CT scan (Somatom Edge vs. Somatom Definition AS+) with an iterative reconstruction algorithm (ADMIRE $v s$. SAFIRE) and detectors (Stellar vs. Ultra Fast Ceramic) with higher performance $(23,24)$. In 10 patients with COVID-19, Agostini et al. found a decrease in CNR between STD and ULD CT acquisitions on lung images for all tissues assessed (16). These differences can be explained by the fact that the authors used the same iterative level (ADMIRE 4) for both CT acquisitions and that the reconstruction kernels used were different from those used in our study. 
Table 4 Diagnostic image quality and confidence level scored by the senior (radiologist 1) and junior (radiologist 2) radiologists on ULD and STD dose chest CT images

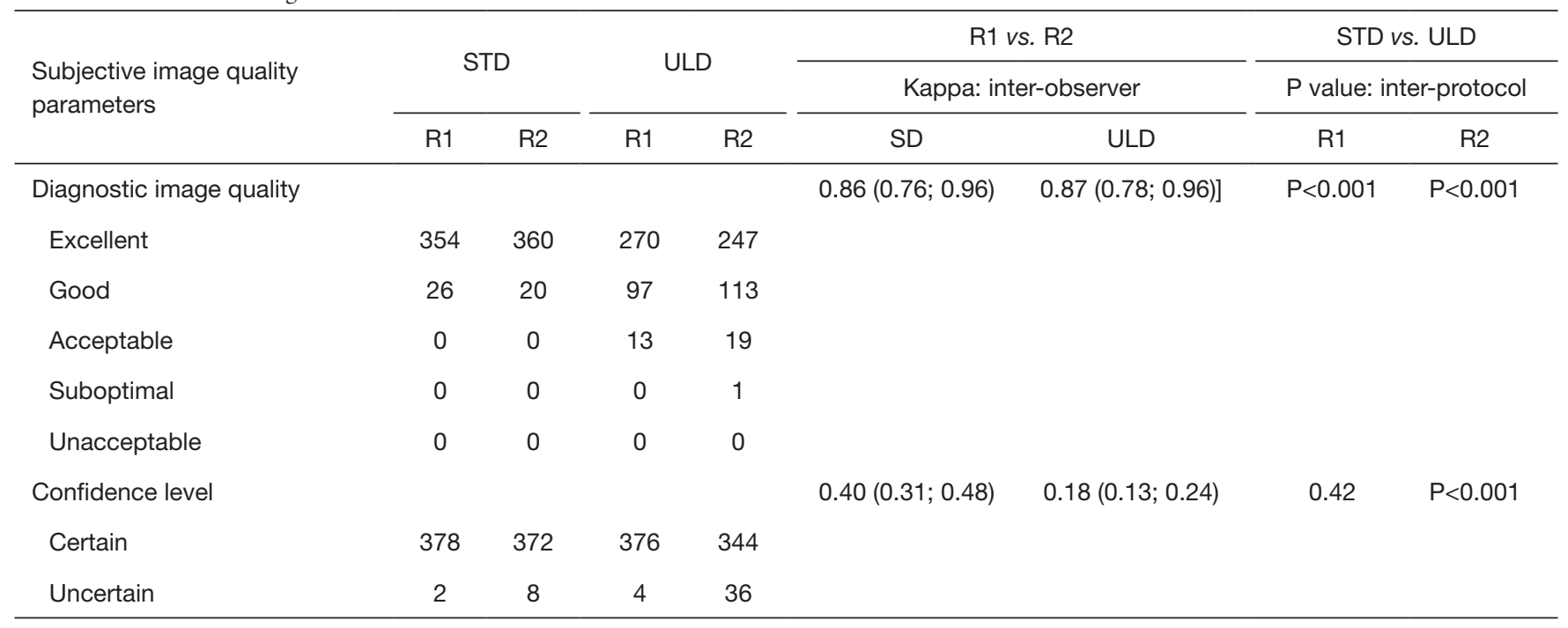

Outcomes of inter observer agreement between readers for both chest CT protocols and inter-protocol comparison for each reader are depicted. A P value lower than 0.05 was considered significant. R1, radiologist 1; R2, radiologist 2; ULD, ultra-low-dose; STD, standard; $\mathrm{CT}$, computed tomography.

These modifications in image quality did not affect the results of the subjective assessment of the diagnostic image quality or diagnostic confidence level. Both radiologists considered the diagnostic image quality of ULD images as "excellent" or "good" in more than $95 \%$ of cases, and a good inter-observer agreement was reported. However, there was a difference between the two radiologists regarding the diagnostic confidence level: the senior radiologist ranked his diagnosis confidence level as "certain" in $99 \%$ of cases and the junior in $91 \%$ of cases. Similar results were found by Macri et al., which suggests that an implicit visual adjustment effect may occur with experience of ULD CT images $(17,18)$. Indeed, R2 was more critical of ULD CT image quality, probably due to his relatively short experience of ULD images, whereas R1 had been working on ULD CT images for several years. This emphasizes the need for specific training on ULD images in order to increase the level of confidence with images of suitable quality for diagnosis.

Our study has certain limitations, the principal one being the lack of a gold standard to assess the sensitivity and specificity of STD and ULD acquisitions. RT-PCR was not available for all patients and all viruses during this unexpected outbreak period. Sensitivity of RTPCR for COVID-19 is around $60-70 \%$ (4) therefore its use may be discussable; however, it is the actual gold standard for COVID-19 diagnosis. Moreover, the severity of pneumonia and the patient's immune status were not evaluated; subtle viral pneumonia could have been missed in immunocompromised patients. In any case, this did not affect the methodology of our study which aimed to compare viral pneumonia pattern detection on STD CT with ULD CT. CT has generally been shown to be more sensitive than RT-PCR (4) but might report false positives with regard to other viral pneumonia $(10,25)$, which is why we chose to study viral patterns and not just specific COVID-19 patterns. Furthermore, this study was performed on a single CT-scan with a specific iterative algorithm, specific intrinsic parameters (such as bowtie filter, filtration, geometry, X-ray tube) and detection chain. Even though the ULD protocol can be adapted on all CT-scans, differences in performance and, therefore, in image quality especially between the various iterative algorithms available on CT-scans, should be considered (24). In addition, the first outcomes obtained with deep learning image reconstruction algorithms depicted more powerful in dealing with noise and a high potential for ULD chest CT. Finally, we did not take into account a possible different inspiratory apnea between STD and ULD acquisitions. 


\section{Conclusions}

The study showed that, compared to STD dose CT, ULD chest CT has comparable diagnostic performance and resulting confidence levels for the detection of viral pneumonia, which could support its implementation in clinical practice during outbreak periods. However, ULD seems to be less effective for studying the interstitial space and should not be used for follow-up when interstitial lung disease such as lung fibrosis, is suspected.

\section{Acknowledgments}

The authors wish to thank all those carers and teams for their professionalism and commitment in looking after patients suspected of COVID-19 infection during this outbreak period. We also thank Teresa Sawyers, Medical Writer at the Laboratoire de Biostatistique, Epidémiologie clinique, Santé Publique, Innovation et Méthodologie (BESPIM) for revising the text.

Funding: None.

\section{Footnote}

Conflicts of Interest: All authors have completed the ICMJE uniform disclosure form (available at http://dx.doi. org/10.21037/qims-20-1176). The authors have no conflicts of interest to declare.

Ethical Statement: The study was conducted in accordance with the Declaration of Helsinki (as revised in 2013) and approved by the institutional review board of the Nîmes University Hospital. The protocol was in accordance with the clinical practices decided in our department to face the pandemic situation. The individual informed consent requirement was waived by the IRB because of the retrospective character of the analysis.

Open Access Statement: This is an Open Access article distributed in accordance with the Creative Commons Attribution-NonCommercial-NoDerivs 4.0 International License (CC BY-NC-ND 4.0), which permits the noncommercial replication and distribution of the article with the strict proviso that no changes or edits are made and the original work is properly cited (including links to both the formal publication through the relevant DOI and the license). See: https://creativecommons.org/licenses/by-nc-nd/4.0/.

\section{References}

1. Galante O, Avni YS, Fuchs L, Ferster OA, Almog Y. Coronavirus NL63-induced adult respiratory distress syndrome. Am J Respir Crit Care Med 2016;193:100-1.

2. Azhar EI, Lanini S, Ippolito G, Zumla A. The Middle East respiratory syndrome coronavirus - a continuing risk to global health security. Adv Exp Med Biol 2017;972:49-60.

3. World Health Organization. Pneumonia of unknown cause-China. 2020 Available online: https://www.who.int/ csr/don/05-january-2020-pneumonia-of-unkown-causechina/en/

4. Fang Y, Zhang H, Xie J, Lin M, Ying L, Pang P, Ji W. Sensitivity of chest CT for COVID-19: comparison to RT-PCR. Radiology 2020;296:E115-7.

5. Ai T, Yang Z, Hou H, Zhan C, Chen C, Lv W, Tao Q, Sun $\mathrm{Z}$, Xia L. Correlation of chest CT and RT-PCR testing in coronavirus disease 2019 (COVID-19) in China: a report of 1014 cases. Radiology 2020;296:E32-40.

6. Chung M, Bernheim A, Mei X, Zhang N, Huang M, Zeng X, Cui J, Xu W, Yang Y, Fayad ZA, Jacobi A, Li K, Li S, Shan H. CT imaging features of 2019 novel coronavirus (2019-nCoV). Radiology 2020;295:202-7.

7. Pan Y, Guan H, Zhou S, Wang Y, Li Q, Zhu T, Hu Q, Xia L. Initial CT findings and temporal changes in patients with the novel coronavirus pneumonia (2019-nCoV): a study of 63 patients in Wuhan, China. Eur Radiol 2020;30:3306-9.

8. Hani C, Trieu NH, Saab I, Dangeard S, Bennani S, Chassagnon G, Revel MP. COVID-19 pneumonia: a review of typical CT findings and differential diagnosis. Diagn Interv Imaging 2020;101:263-8.

9. Li J, Long X, Wang X, Fang F, Lv X, Zhang D, Sun Y, Hu $\mathrm{S}$, Lin Z, Xiong N. Radiology indispensable for tracking COVID-19. Diagn Interv Imaging 2021;102:69-75.

10. Bai HX, Hsieh B, Xiong Z, Halsey K, Choi JW, Tran TML, Pan I, Shi LB, Wang DC, Mei J, Jiang XL, Zeng QH, Egglin TK, Hu PF, Agarwal S, Xie FF, Li S, Healey T, Atalay MK, Liao WH. Performance of radiologists in differentiating COVID-19 from non-COVID-19 viral pneumonia at chest CT. Radiology 2020;296:E46-54.

11. Koo HJ, Lim S, Choe J, Choi SH, Sung H, Do KH. Radiographic and CT Features of Viral Pneumonia. Radiographics 2018;38:719-39.

12. Pan F, Ye T, Sun P, Gui S, Liang B, Li L, Zheng D, Wang J, Hesketh RL, Yiang L, Zheng C. Time course of lung changes on chest CT during recovery from 2019 
novel coronavirus (COVID-19) pneumonia. Radiology 2020;295:715-21.

13. Yuan $M$, Yin W, Tao Z, Tan W, Hu Y. Association of radiologic findings with mortality of patients infected with 2019 novel coronavirus in Wuhan, China. PLoS One 2020;15:e0230548.

14. Herpe G, Naudin M, Léderlin M, Enikeeva F, Boumendil O, Cassagnes L, Cavet M, Chaumoitre K, Feuerstein P, Fitton I, Flory V, Freitag CA, Gaubert JY, Gregory J, Nivet H, Ohana M, Petit I, Sans N, Wagner M, Guillevin R, Saulnier PJ, Bartoli JM, Tasu JP, Beregi JP. COVID-19 impact assessment on the French radiological centers: a nationwide survey. Eur Radiol 2020;30:6537-44.

15. Kang Z, Li X, Zhou S. Recommendation of low-dose CT in the detection and management of COVID-2019. Eur Radiol 2020;30:4356-7.

16. Agostini A, Floridi C, Borgheresi A, Badaloni M, Esposto Pirani P, Terilli F, Ottaviani L, Giovagnoni A. Proposal of a low-dose, long-pitch, dual-source chest CT protocol on third-generation dual-source CT using a tin filter for spectral shaping at $100 \mathrm{kVp}$ for CoronaVirus Disease 2019 (COVID-19) patients: a feasibility study. Radiol Med 2020;125:365-73.

17. Macri F, Greffier J, Pereira F, Rosa AC, Khasanova E, Claret PG, Larbi A, Gualdi G, Beregi JP. Value of ultralow-dose chest CT with iterative reconstruction for selected emergency room patients with acute dyspnea. Eur J Radiol 2016;85:1637-44.

18. Macri F, Greffier J, Khasanova E, Claret PG, Bastide S, Larbi A, Bobbia X, Pereira FR, De la Coussaye JE, Beregi JP. Minor blunt thoracic trauma in the emergency

Cite this article as: Greffier J, Hoballah A, Sadate A, de Oliveira F, Claret PG, de Forges H, Loubet P, Mauboussin JM, Hamard A, Beregi JP, Frandon J. Ultra-lowdose chest $\mathrm{CT}$ performance for the detection of viral pneumonia patterns during the COVID-19 outbreak period: a monocentric experience. Quant Imaging Med Surg 2021;11(7):3190-3199. doi: 10.21037/qims-20-1176 department: sensitivity and specificity of chest ultralowdose computed tomography compared with conventional radiography. Ann Emerg Med 2019;73:665-70.

19. Deak PD, Smal Y, Kalender WA. Multisection CT protocols: sex- and age-specific conversion factors used to determine effective dose from dose-length product. Radiology 2010;257:158-66.

20. Rossi SE, Erasmus JJ, Volpacchio M, Franquet T, Castiglioni T, McAdams HP. "Crazy-paving" pattern at thin-section CT of the lungs: radiologic-pathologic overview. Radiographics 2003;23:1509-19.

21. Greffier J, Macri F, Larbi A, Fernandez A, Khasanova E, Pereira F, Mekkaoui C, Beregi JP. Dose reduction with iterative reconstruction: optimization of CT protocols in clinical practice. Diagn Interv Imaging 2015;96:477-86.

22. Greffier J, Larbi A, Frandon J, Moliner G, Beregi JP, Pereira F. Comparison of noise-magnitude and noisetexture across two generations of iterative reconstruction algorithms from three manufacturers. Diagn Interv Imaging 2019;100:401-10.

23. Greffier J, Frandon J, Larbi A, Om D, Beregi JP, Pereira F. Noise assessment across two generations of iterative reconstruction algorithms of three manufacturers using bone reconstruction kernel. Diagn Interv Imaging 2019;100:763-70.

24. Greffier J, Frandon J, Larbi A, Beregi JP, Pereira F. CT iterative reconstruction algorithms: a task-based image quality assessment. Eur Radiol 2020;30:487-500.

25. Dalglish SL. COVID-19 gives the lie to global health expertise. Lancet 2020;395:1189. 